\title{
Diagnosis Structural Components Prelaunch Emotional States in Sportsman Single Combat
}

\author{
Andrey Vyacheslavovich Kernas ${ }^{1}$ \\ ${ }^{1}$ Postgraduate the Institute of Psychology South Ukrainian National Pedagogical University named by K.D. \\ Ushinskogo, Ukraine \\ Correspondence: Andrey Vyacheslavovich Kernas, Postgraduate the Institute of Psychology South Ukrainian \\ National Pedagogical University named by K. D. Ushinskogo, Ukraine. E-mail: bastion_odessa@mail.ru
}

\author{
Received: May 23, 2013 Accepted: August 25, 2014 Online Published: September 29, 2014 \\ doi:10.5539/ass.v10n19p30 URL: http://dx.doi.org/10.5539/ass.v10n19p30
}

\begin{abstract}
Theoretical and practical development and validation aspects of psychodiagnostic method aimed at diagnosing structural components of prelaunch emotional athletes engaged in different kinds of combat sports are discussed. Development and testing of the above psychodiagnostic method are carried out in the course of the dissertation research. The sample consisted of athletes that representing various types and directions of combat sports.
\end{abstract}

Keywords: sports psychodiagnosis, prelaunch emotional state, structural components, athletes

\section{Introduction}

Method of diagnostics the structural components of prelaunch emotional states in combat sports athletes, engaged in various kinds of martial arts was developed by us in the course of work, aimed at studying the phenomenological nature prelaunch emotional states: combat readiness, fever and apathy, and isolating series components included in their structure: aggressive, thinking, motivational, physical, willed.

In turn, the disclosure of their psychological content has allowed substantiating theoretically degree of expressed and proportional ratio display characteristics typical of the experience the athlete-single combat one or another prestart emotional state, which was put in the fundamental basis of psycho-diagnostic tools created by us (Kernas, 2012).

The author's rights for the methodology were registered (The Certificate No. 54849 from 16.05.2014).

In developing this psychodiagnostic method, we carried out the work with scientific and methodological literature containing information on the studied area (Alekseev, 1979; Bochelyuk, 2007; Evseev, 2005; Ilyin, 2009; Kadochnikov, 2006; Korolchuk, 2009; Chernikova, 1962).

\section{Discussion}

Thus, we stated the following.

At present in psychology of sport three pre-sport emotional states as military readiness, fever and apathy are distinguished. In this respect the large majority of specialists A. C. Puni (1973), O. A. Chernikova (1962), A. V. Alekseev (1979), A. A. Kadochnikov (2003) expresses consent in the views at that the pre-start emotional state of military readiness is the optimal form promoting the successful execution of the sport activity. At the same time the pre-start emotional states of fever and apathy are defined by the specialists as such one, which negatively effect on the adaptation of sportsmen to the conditions of the executed professional activity, and as a result of it its effectiveness.

In his turn, E. P. Ilyin (2008a) indicates that in practice it is not always so. Furthermore, E. P. Ilyin explains that the threshold of appearance of these states at different people is not similar. People of innervate type have much more strong the pre-start emotional excitement than people of inhibitory type. Consequently, this level of excitement, which for the last one will be close to "fever", while for the first ones will be ordinary pre-start state. Therefore, it is necessary to take into account the individual peculiarities of the emotional excitement and responsiveness of different people (Ilyin, 2008a).

In our view, one of the variants of solution of the problem sounded by the author can become division of the pre-start emotional states on the structural elements they are constituted from, at further consideration of which 
the logical conditionality of the positive or negative effect on the adaptation of the sportsmen in dependence on the extent of their expressiveness and proportional relation are traced.

The study of phenomenological nature conducted by us allowed separating the range of components, which enter into the structure: military readiness, fever and apathy. In its turn, the reveal of their psychological contents allowed theoretically substantiate the extent of expressiveness and proportional relation of the evident characteristic peculiarities, inherent to feeling of this or those pre-start emotional state by the sportsman-combat athlete.

On the assumption that the aim of our work is the development of the system, directed at the psychological correction of the negative pre-start emotional states and formation of the pre-start emotional state of military readiness, optimally promoting to adaptation of sportsman and successful implementation of sport activity, we consider necessary to pay attention to that the supposed advanced result testifying about practical effectiveness of the complex methodology offered by us, and its successful approbation in the sport groups must have become confirmation in the form of received actual data, objectively testifying about enhancement of military readiness due to reduction of fever or apathy. Only in this case we can consider the conducted practical work to be successful.

Thus, we consider it appropriate to focus attention on the diagnostics of structural components, according to its psychological meaning, the extent of expressiveness and proportional relation optimally corresponding to feeling the pre-start emotional state of military readiness by the sportsman.

As it was established by us, the structure of pre-start emotional states includes the following components: physical, intellectual, aggressive and conative.

Let's consider one of the specified components in separate.

The physical component. The readiness of the sportsman to the start, physical loadings (trainings) is the readiness in the shortest time to pass from the rest to work, and reach the optimal working capacity, and pass from one type or level of intensiveness of work to others, providing the required quality of physical loading. The readiness is one of the characteristics of working capacity, as in many cases it is important not only to execute work (exercise) of the certain intensiveness and (or) duration, but also in time or possible to begin it earlier (Korolchuk, 2009). In sportsman-combat athlete the physical component is revealed in the feelings corresponding physical qualities, the state of neuromuscular apparatus, different organs and systems.

Intellectual component. The thinking in the modern understanding is defined as the highest form of active reflection of the objective reality that is formed in the appropriate, indirect and generalized consciousness of a man, the consciousness of the connections and relations of the subjects and phenomena, in solution of the problem situation, creation of the new ideas, prognosis of the possible situations and actions adequate to them (Korolchuk, 2009). Thus, the intellectual component represents in essence the plan and programme of activity, which the sportsman-combat athlete focuses at. In the opinion of E. P. Ilyin the peculiar role at preparation to competitions belongs to the analytical thinking, as one of the parties of intellect of the sportsman. At the same time Ilyin indicates that in sport type of combat sport the intellect is implemented more frequently in the tactical and operative thinking, at that paying attention to that in literature devoted to the psychology of sport, there is identification of these types of thinking that is not right at all. Although, the operative thinking is close by its characteristics to the tactical thinking: the first and the second one have indirect and figuratively-efficient character, and are connected with prognosticating of the future situation, however, the tactical thinking is connected more with preparation to activity with the development of its plan, while the operative thinking with implementation of this plan with urgent putting of corrections into it, if something prevents to implementation of the forethought. It is possible to say that the operative thinking is the means of implementation of the tactic thinking (Ilyin, 2008b).

Aggressive component. Aggression is the behaviour, at which the purpose of to doing physical and moral damage to other people or limiting their desires is pursued. From this point of view, the sport is aggressive by its competitive essence, as the sportsmen aspire to strangulate aspiration of others to victory (Ilyin, 2008c). At sportsman-combat athlete the aggressive component displays itself in the extent of expressiveness of aggression and its capability to implementation of the rational appropriate control of the own aggression. As J. Kretti indicates in many types of sport the participants are required to reveal aggressiveness within the framework of existing rules and conditions of activity (Cretti, 2007). At the same time it is known, that badly controlled aggression not rarely turns against the individual, destructively affecting both on the state of his health, and on the results of the activity done by him. In respect to the activity in the sport types of combat sports in our view the controlled game aggression fits optimally. The detailed description of the peculiarities inherent to this 
phenomenon is stated by E. Fromm in his work "The Types of Aggression", from which it is follows that the game aggression is necessary in the educational training for mastery, skilfulness and response quickness. It has no any destructive purpose and no negative motivations (anger, hatred) (Fromm, 2005). In the scientific practice, devoted to consideration of this issue, we find the opinion about that such type of good aggression is adaptive, directed at socially appropriate self-assertiveness in the conditions of competition (Platonova, 2004).

Conative component. As A. Y. Chebykin (2007), Todorova (2007), E. P. Ilyin (2009), A. G. Maklakov (2006) indicates, the interrelation of the motor and emotional conative activities is the indispensable component of the sportsmen's development, at that they focus attention at the fact that the mechanisms of interrelation of emotions and will are more frequently traced at the level of regulatory function, psychologically connected with activation, mobilization and quieting. V. I. Bochelyuk and O. A. Cherepakhina draw attention to that presently in sport psychology the following qualities of conative preparation of the sportsman are separated as appropriateness (objective aspiration to achievement of the prospective purpose), decisiveness and courage (tendency to the reasonable risk in combination with the timely understanding of the decisions), persistence (capability to mobilization of the functional reserves, activeness in achievement of the set aims and overcome of the obstacles), self-possession and self-control (mind lightness, ability to control over own thoughts and actions in the conditions of the emotional tension), independence and initiative (Bochelyuk \& Cherepakhina, 2007).

\section{Results}

Thus, after carried out the generalized analysis we isolated universally recognized in the scientific field of knowledge patterns and descriptive characteristics we studied phenomenological manifestations. Which in turn amounted fundamental and basic fundamentals, based on which we had designed a plan to build a common psychodiagnostic method.

In developing the methodology we were guided to create effective psychodiagnostic tools that allow maximum objectivity and accuracy to determine the level of manifestation of structural components, prestart emotional state athlete single combat.

The next stage of our work on the diagnostic procedure was to undertake primary trial testing in three sports groups cultivating sports martial arts.

In testing was attended by 52 single combat athletes.

Also on our initiative was conducted comprehensive counseling discussions with a team of professional psychologists, aimed at assessing the degree of clarity of instructions and the wording of the proposed statements, as well as in determining the content (face) validity psychodiagnostic method.

Positive results obtained by us in the end of work done, convinced us psychodiagnostic method the need to maintain in its original incarnation unchanged. After that, we performed a large-scale work aimed to conduct psychodiagnostic procedures in sports clubs, cultivating different kinds of martial arts. Just testing psychodiagnostic method participated 148 athletes single combat. This led to the comparison of the data identified methods of observation, discussions and questionnaires, we can draw conclusions about the validity of the proposed psychodiagnostic method. Consequently, we can recommend it for use in sporting groups, cultivating different kinds of martial arts.

Instructions, stimulus material and the keys to method are set out below.

Method of diagnostics the structural components of the emotional state prestart

Full name

Age

Sports kind

Experience of employment

Sporting achievements

Date of completion

Here is methodology whose aim is identify the degree of correlation and the structural components of your prestart emotional condition arising when you implement a training-preparatory and competitive activities. Respond to each statement to select one of four possible answers: a) - "yes", and b) more "yes" than "no", c) more "no" than "yes", d) - "no . "Select one that is closest to you. Try to give answers quickly, without thinking about the details. Let generalized answers, focusing on its own experience playing sports. 


\section{Aggressive component}

1. Participating in competitions, I often lose control of myself and I cannot resist not to break the rules.

a) yes

b) no

c) rather yes

g) rather no

d) is difficult to answer

2. As a rule, I do not feel personal dislike to my opponents in competition.

a) yes

b) no

c) rather yes

g) rather no

d) is difficult to answer

3. I am always ready to shake hands with to your opponent after Sport Wrestling, regardless of whether in whose favor it over.

a) yes

b) no

c) rather yes

g) rather no

d) is difficult to answer

4. I think that sports martial arts - a sport of gentlemen, which are not allowed victories in violation of the rules, with the connivance of refereeing.
a) yes
b) no
c) rather yes
g) rather no
d) is difficult to answer

5. I always during a speech in competition is able to control the manifestations of my aggression.

a) yes

b) no

c) rather yes

g) rather no

d) is difficult to answer

6. I capable to cause a controlled game aggression.

a) yes

b) no

c) rather yes

g) rather no

d) is difficult to answer

\section{Intellective component}

1. As a rule, I try to use a known me information about the strengths and weaknesses of my opponent, in selecting complex technical and tactical actions for a specific fight (battle).

a) yes 
b) no

c) rather yes

g) rather no

d) is difficult to answer

2. In most cases, I'm working on proactive actions of my opponents.

a) yes

b) no

c) rather yes

g) rather no

d) is difficult to answer

3. I find it difficult to adapt to enemy action, ever changing tactics of during the fight (battle).

a) yes

b) no

c) rather yes

g) rather no

d) is difficult to answer

4. I can easily adjust to enemy action - left-handed, using effective methods of counteraction.

a) yes

b) no

c) rather yes

g) rather no

d) is difficult to answer

5. I find myself universal fighter (wrestler), capable the instantaneous assessment of the situation and choosing the most effective technical and tactical actions.

a) yes

b) no

c) rather yes

g) rather no

d) is difficult to answer

6. I find myself flexible fighter (wrestler) able to make timely adjustments depending on the changing situation of wrestling.

a) yes

b) no

c) rather yes

g) rather no

d) is difficult to answer

Physical component

1. I think that at the moment I am in the best physical shape.

a) yes

b) no

c) rather yes

g) rather no

d) is difficult to answer 
2. Usually during carrying out competition, I am tired quickly.

a) yes

b) no

c) rather yes

g) rather no

d) is difficult to answer

3. I see the actions of the enemy, but does not physically have time to respond to them.

a) yes

b) no

c) rather yes

g) rather no

d) is difficult to answer

4. In the context of prolonged opposition competitive struggle, I often do not have enough physical strength to carry out decisive reception.
a) yes
b) no
c) rather yes
g) rather no
d) is difficult to answer

5. Usually with endurance I do not have problems.

a) yes

b) no

c) rather yes

g) rather no

d) is difficult to answer

6. As a rule, I'm starting to feel keenly shortage of physical condition in the first half of the fight (battle).

a) yes

b) no

c) rather yes

g) rather no

d) is difficult to answer

\section{Volitional component}

1. Even having received an injury, I am ready to continue the fight for the victory.

a) yes

b) no

c) rather yes

g) rather no

d) is difficult to answer

2. Usually I do not pay attention to uncomplimentary comments and shouting fans in my direction.

a) yes

b) no

c) rather yes

g) rather no 
d) is difficult to answer

3. Taking part in the competition, I am determined from the beginning to the end to fight for victory.

a) yes

b) no

c) rather yes

g) rather no

d) is difficult to answer

4. I prefer abandon to continue the fight, seeing that the enemy is much more experienced and stronger than me.

a) yes

b) no

c) rather yes

g) rather no

d) is difficult to answer

5. Even if not explicitly objective judging against me, I will make every effort to finish the fight (battle) in my favor.

a) yes

b) no

c) rather yes

g) rather no

d) is difficult to answer

6. As a rule, I continue the fight (battle) to the end, regardless of the pain and fatigue.

a) yes

b) no

c) rather yes

g) rather no

d) is difficult to answer

\section{Motivational component}

1. I am willing to spare no effort to go to the achievement of sporting purposes.

a) yes

b) no

c) rather yes

g) rather no

d) is difficult to answer

2. I will make all its potential in order to justify the confidence of the coach (coaching and teaching staff) believe in me.
a) yes
b) no
c) rather yes
g) rather no
d) is difficult to answer
3. In sports, I set itself the goal to achieve fame and recognition.
a) yes
b) no 
c) rather yes

g) rather no

d) is difficult to answer

4. Going in for sports, I set myself the goal to improve financial position.
a) yes
b) no
c) rather yes
g) rather no
d) is difficult to answer

\section{Keys to the calculation of the structural components of the prelaunch emotional state athlete single combat}

To obtain the test results to summarize all relevant key table, the numerical values multiplied by a factor of significance and compare them with the proposed limits of the test.

Aggressive component

\begin{tabular}{ccccccc}
\hline Questions & $\mathrm{a}$ & $\mathrm{b}$ & $\mathrm{c}$ & $\mathrm{d}$ & $\mathrm{e}$ & Coefficient of significance \\
\hline 1 & -2 & 2 & -1 & 1 & 0 & 2 \\
2 & 2 & -2 & 1 & -1 & 0 & 1 \\
3 & 2 & -2 & 1 & -1 & 0 & 1 \\
4 & 2 & -2 & 1 & -1 & 0 & 1 \\
5 & 2 & -2 & 1 & -1 & 0 & 1 \\
6 & 2 & -2 & 1 & -1 & 0 & 3 \\
\hline
\end{tabular}

\begin{tabular}{ccc}
\hline Poor & Average & Good \\
\hline$-18-6$ & $-6+6$ & $+6+18$ \\
\hline
\end{tabular}

Intellectual component

\begin{tabular}{ccccccc}
\hline Questions & $\mathrm{a}$ & $\mathrm{b}$ & $\mathrm{c}$ & $\mathrm{d}$ & $\mathrm{e}$ & Coefficient of significance \\
\hline 1 & 2 & -2 & 1 & -1 & 0 & 2 \\
2 & 2 & -2 & 1 & -1 & 0 & 3 \\
3 & -2 & 2 & -1 & 1 & 0 & 3 \\
4 & 2 & -2 & 1 & -1 & 0 & 1 \\
5 & 2 & -2 & 1 & -1 & 0 & 3 \\
6 & 2 & -2 & 1 & -1 & 0 & 1 \\
\hline
\end{tabular}

\begin{tabular}{ccc}
\hline Poor & Average & Good \\
\hline$-26-14$ & $-14+14$ & $+14+26$ \\
\hline
\end{tabular}

Physical component

\begin{tabular}{ccccccc}
\hline Questions & A & b & c & d & e & Coefficient of significance \\
\hline 1 & 2 & -2 & 1 & -1 & 0 & 1 \\
2 & -2 & 2 & -1 & 1 & 0 & 3 \\
3 & -2 & 2 & -1 & 1 & 0 & 1 \\
4 & -2 & 2 & -1 & 1 & 0 & 3 \\
5 & -2 & -2 & 1 & -1 & 0 & 1 \\
6 & -2 & -2 & -1 & 1 & 0 & 3 \\
\hline
\end{tabular}

\begin{tabular}{ccc}
\hline Poor & Average & Good \\
\hline$-24-12$ & $-12+12$ & $+12+24$ \\
\hline
\end{tabular}

Volitional component

\begin{tabular}{ccccccc}
\hline Questions & $\mathrm{a}$ & $\mathrm{b}$ & $\mathrm{c}$ & $\mathrm{d}$ & $\mathrm{e}$ & Coefficient of significance \\
\hline 1 & 2 & -2 & 1 & -1 & 0 & 3 \\
2 & 2 & -2 & 1 & -1 & 0 & 1 \\
3 & 2 & -2 & 1 & -1 & 0 & 1 \\
4 & -2 & 2 & -1 & 1 & 0 & 3 \\
5 & 2 & -2 & 1 & -1 & 0 & 1 \\
6 & 2 & -2 & 1 & -1 & 0 & 2 \\
\hline
\end{tabular}

\begin{tabular}{ccc}
\hline Poor & Average & Good \\
\hline$-22-11$ & $-11+11$ & $+11+22$ \\
\hline
\end{tabular}

Motivational component

\begin{tabular}{|c|c|c|c|c|c|c|c|c|c|}
\hline Questions & $\mathrm{a}$ & $\mathrm{b}$ & $\mathrm{c}$ & $\mathrm{d}$ & $\mathrm{e}$ & Coefficient of significance & & & \\
\hline 1 & 2 & -2 & 1 & -1 & 0 & 1 & & & \\
\hline 2 & 2 & -2 & 1 & -1 & 0 & 2 & Poor & Average & Good \\
\hline 3 & 2 & -2 & 1 & -1 & 0 & 2 & $-12-6$ & $-6+6$ & $+6+12$ \\
\hline 4 & 2 & -2 & 1 & -1 & 0 & 1 & & & \\
\hline
\end{tabular}




\section{References}

Alekseev, A. V. (1979). The psychology of sport of the highest achievements. Moscow: The Physical culture and sport.

Bochelyuk, V. I., \& Cherepakhina, O. A. (2007). The psychology of sport. Kyiv: The centre of the study materials.

Chebykin, A. Y. (2007). The emotional-volitional sphere of the students, engaged in the different types of the physical culture. Odessa: Edited by Cherkasov.

Chernikova, O. A. (1962). The psychological peculiarities of the influence of emotions on the sportsman's activity. The digest of the scientific articles "The problems of the sport psychology", 6, 198-210.

Cretti, J. (2007). The aggressiveness in sport. In The Psychology of Sport: Reading-Book (pp. 147-159). Moscow, Minsk: AST, Harvest.

Dubrovskii, V. I. (2005). The physiology of sport. Moscow: The humanitarian publishing centre "Vlados".

Evseev, Y. I. (2005). The physical culture. Rostov-on-Don: Phoenix.

Fromm, E. (2005). The type of aggression. In The Psychology of the Human Aggressiveness: Reading-Book (pp. 354-427). Moscow, Minsk: AST, Harvest.

Ilyin, E. P. (1962). The change of movement coordination at the pupils of the secondary school age under influence of the different emotional states. The problems of the psychology of sport, 6, 212-218.

Ilyin, E. P. (2008a). The emotions and feelings. Saint-Petersburg: The Publishing House "Peter".

Ilyin, E. P. (2008b). The motivation and motives. Saint-Petersburg: The Publishing House "Peter".

Ilyin, E. P. (2008c). The psychology of sport. Saint-Petersburg: The Publishing House "Peter".

Ilyin, E. P. (2009). The psychology of will. Saint-Petersburg: The Publishing House "Peter".

Kadochnikov, A. A. (2003). The psychological training to the foot to foot fight. Rostov-on-Don: Phoenix.

Kadochnikov, A. A. (2006). Ready to the fight! Stress resistance in foot to foot fight. Rostov-on-Don: Phoenix.

Kernas, A. V. (2012, 18th-19th). The psychological peculiarities of adaptation of the young sportsmen-combatants to the stressogenic conditions of the competitive activity. Munich, Germany. Materials of the II International research and practice conference, 2, 546-548.

Korolchuk, M. S. (2009). The psychophysiology activity. Kyiv: Elga, Nika-Centre.

Maklakov, A. G. (2009). The general psychology. Saint-Petersburg: The Publishing House "Peter".

Puni, A. C. (1973). Some psychological issues of readiness to the competitions in sport. Leningrad: GDOIFK.

Platonova, N. M. (Ed.). (2004). Aggression at children and teenagers. Saint-Petersburg: Rech.

\section{Copyrights}

Copyright for this article is retained by the author(s), with first publication rights granted to the journal.

This is an open-access article distributed under the terms and conditions of the Creative Commons Attribution license (http://creativecommons.org/licenses/by/3.0/). 